\title{
ZEROS OF SOLUTIONS OF A SECOND ORDER NONLINEAR DIFFERENTIAL INEQUALITY
}

\author{
FU-HSIANG WONG
}

(Communicated by Kenneth R. Meyer)

\begin{abstract}
Under suitable assumptions on $r, g$, and $F$, we show that every zero of a solution of the nonlinear differential inequality
\end{abstract}

$$
\left(r(t) y^{\prime}(t)\right)^{\prime}+g(t) F(y(t)) \leq 0(\geq 0)
$$

is simple.

Recently, Kwong [1] proved the following very interesting result:

Theorem A. If $y(t)$ is positive (negative) in an interval $(a, b)$ with $y(a)=0$ or $y(b)=0$ and it satisfies the inequality

$$
y^{\prime \prime}(t)+f(t) y^{\prime}(t)+g(t) y(t) \leq 0(\geq 0) \text { in }(a, b),
$$

where $f$ and $g$ are continuous functions, then $y^{\prime}(a) \neq 0$ or $y^{\prime}(b) \neq 0$; that is, the given zeros of the solution are simple.

Kwong's proof is based on the formula of variation of constants.

The purpose of this article is to extend Theorem A to a nonlinear case by using the following LaSalle inequality [2]:

\section{Theorem B. Let}

$\left(C_{1}\right) \quad \bar{F} \in C([0, c] ;[0, \infty))$ be positive and nondecreasing on $(0, c)$, for some $c>0$,

$\left(C_{2}\right) \quad h \in L^{1}(\mathbf{R} ;[0, \infty))$,

$\left(C_{3}\right) \quad y \in C([a, b] ;[0, c))$.

Then the inequalities

$$
y(t) \leq \int_{a}^{t} h(s) \bar{F}(y(s)) d s \quad \text { for } t \in[a, b]
$$

Received by the editors December 5, 1989.

1980 Mathematics Subject Classification (1985 Revision). Primary 34C10.

Key words and phrases. Simple zero, LaSalle's inequality. 
and

$$
y(t) \leq \int_{t}^{b} h(s) \bar{F}(y(s)) d s \quad \text { for } t \in[a, b]
$$

imply that

$$
\int_{0}^{y(t)} \frac{d s}{\bar{F}(s)} \leq \int_{a}^{t} h(s) d s \quad \text { for } t \in[a, b]
$$

and

$$
\int_{0}^{y(t)} \frac{d s}{\bar{F}(s)} \leq \int_{t}^{b} h(s) d s \quad \text { for } t \in[a, b],
$$

respectively. In addition, if

$$
\text { ( } \left.C_{4}\right) \quad \int_{0}^{c} \frac{d s}{\bar{F}(s)}=\infty \text {, then } y(t) \equiv 0 \text { on }[a, b] \text {. }
$$

Theorem 1. Assume that

$\left(C_{5}\right) \quad r \in L^{1}([a, b] ;(0, \infty))$ and $g \in L^{1}([a, b] ; \mathbf{R})$,

( $\left.C_{6}\right) \quad F \in L^{1}(\mathbf{R} ; \mathbf{R})$ and there exist a positive constant $c$ and $a$ function $\bar{F}$ satisfying $\left(\mathrm{C}_{1}\right),\left(\mathrm{C}_{4}\right)$, and $|F(y)| \leq \bar{F}(|y|)$ for all $y \in(-c, c)$.

Suppose that $y(t)$ is positive (negative) in the interval $(a, b)$ with $y(a)=0$ or $y(b)=0$ and it satisfies

$$
\left(r(t) y^{\prime}(t)\right)^{\prime}+g(t) F(y(t)) \leq 0(\geq 0) .
$$

Then $y^{\prime}(a) \neq 0$ or $y^{\prime}(b) \neq 0$.

Proof. We prove only the case $y(a)=0$. Suppose to the contrary that $y^{\prime}(a)=$ 0 .

Case (1). Suppose that $y(t)>0$ on $(a, b)$. It follows from (6) that

$$
r(t) y^{\prime}(t) \leq-\int_{a}^{t} g(s) F(y(s)) d s, \quad \text { for } t \in(a, b),
$$

which implies

$$
y(t) \leq-\int_{a}^{t}\left\{\frac{1}{r(s)} \int_{a}^{s} g(u) F(y(u)) d u\right\} d s
$$

Then

$$
\begin{aligned}
y(t) & \leq \int_{a}^{t} m|g(s)||F(y(s))| d s \\
& \leq \int_{a}^{t} m|g(s)||\bar{F}(y(s))| d s, \text { for } t \in[a, d]
\end{aligned}
$$


where $m:=\int_{a}^{b} \frac{d s}{r(s)}$ and $d \in(a, b]$ such that $|y(t)|<c$ for $t \in[a, d]$. Hence, by LaSalle's inequality, we see that $y(t) \equiv 0$ on $[a, d]$. This contradicts the hypothesis $y(t)>0$ on $(a, b)$.

Case (2). Suppose that $y(t)>0$ on $(a, b)$. It follows from (6) that

$$
r(t) y^{\prime}(t) \geq-\int_{a}^{t} g(s) F(y(s)) d s, \quad \text { for } t \in(a, b),
$$

which implies that

$$
y(t) \geq-\int_{a}^{t}\left\{\frac{1}{r(s)} \int_{a}^{s} g(u) F(y(u)) d u\right\} d s .
$$

Then

$$
\begin{aligned}
|y|(t) \mid & \leq \int_{a}^{t} m|g(s)||F(y(s))| d s \\
& \leq \int_{a}^{t} m|g(s)||\bar{F}(y(s))| d s, \quad \text { for } t \in[a, d],
\end{aligned}
$$

where $m$ and $d$ are defined as in Case (1). Hence, by LaSalle's inequality, we see that $y(t) \equiv 0$ on $[a, d]$. This contradicts the hypothesis $y(t)<0$ on $(a, b)$.

Theorem 2. Assume that

$\left(C_{7}\right) \quad r \in C([a, b] ;(0, \infty))$ and $g \in C([a, b] ; \mathbf{R})$,

$\left(C_{8}\right) \quad F \in L^{1}(\mathbf{R} ; \mathbf{R})$ such that $y F(y)>0$ on $(-c, c)-\{0\}$ for some $c>0$, and

$$
\int_{0}^{c} \frac{d s}{\left(\int_{0}^{s} F(s) d s\right)^{1 / 2}}=\infty
$$

Suppose that $y(t)$ is positive (negative) in the interval $(a, b)$ with $y(a)$ or $y(b)=0$ and it satisfies (6). Then $y^{\prime}(a) \neq 0$ or $y^{\prime}(b) \neq 0$.

Proof. Using the classical Liouville transformation, we can always get rid of the coefficient $r(t)$. So we may assume without loss of generality that the inequality we are studying is

$$
y^{\prime \prime}(t)+g(t) F(y(t)) \leq 0 .
$$

Let $C>0$ be a large constant, larger than the maximum of $|g(t)|$ over $[a, b]$. Then

$$
y^{\prime \prime}(t)-C F(y(t)) \leq-(C+|g(t)|) F(y(t)) \leq 0,
$$

at least in a neighborhood of $a$ when $|y(t)|$ is still less than $c$. We can now work with (7), which has a constant coefficient $C$. Multiplying (8) by $y^{\prime}(t)$ and integrating it from $a$ to $t$ (near $a$ ), we obtain that

$$
y^{\prime 2}(t) \leq 2 c \int_{0}^{y(t)} F(s) d s,
$$


which implies that

$$
y^{\prime}(t) \leq\left\{2 c \int_{0}^{y(t)} F(s) d s\right\}^{1 / 2} .
$$

Integrating the above inequality from $a$ to $t$ (near $a$ ), one obtains that

$$
y(t) \leq \sqrt{2 c} \int_{a}^{t}\left\{\int_{0}^{y(s)} F(u) d u\right\}^{1 / 2} d s .
$$

Hence, by LaSalle's inequality, we see that $y(t) \equiv 0$ in a neighborhood of $a$. This contradicts the hypothesis $y(t)>0$ on $(a, b)$.

\section{ACKNOWLEDGMENT}

The author would like to express his gratitude to the referee for a number of helpful suggestions.

\section{REFERENCES}

1. M. K. Kwong, Uniqueness of positive solutions of $\Delta u-u+u^{p}=0$ in $\mathbf{R}^{n}$, Arch. Rational Mech. Anal. 105 (1989), 243-266.

2. J. P. LaSalle, Uniqueness theorems and successive approximations, Ann. of Math. 50 (1949), 722-730.

Department of Mathematics, National Central University, Chung-Li, Taiwan, RePUBLIC OF CHINA 